\title{
From the Breast to the Bowel: An Unconventional Metastatic Presentation
}

\author{
Mounika Gangireddy ${ }^{1}$, Isha Shrimanker ${ }^{1}$, Sandy Saintelia ${ }^{1}$, Janet Gomez ${ }^{1}$, Kathryn A. Peroutka ${ }^{2}$ \\ 1. Internal Medicine, UPMC Pinnacle, Harrisburg, USA 2. Hematology-Oncology, Andrews and Patel Associates, \\ Harrisburg, USA
}

Corresponding author: Isha Shrimanker, ims18919@gmail.com

\begin{abstract}
Breast cancer is the most common cancer in women. The common sites of metastasis include the lungs, liver, and, infrequently, the gastrointestinal (GI) tract. A 72-year-old Caucasian female presented to the hospital with nausea and vomiting, diarrhea, intermittent abdominal pain, and unintentional weight loss. She had had a past medical history of bilateral lobular breast carcinoma and severe iron-deficiency anemia treated with iron transfusions. On arrival, the examination was significant for hypotension and pallor. Laboratory investigations revealed abnormal liver enzymes and raised tumor markers Ca-125 and carcinoembryonic antigen. Imaging studies established a diagnosis of distal small bowel obstruction. The surgical intervention showed the presence of a small bowel tumor, the biopsy findings of which were consistent with metastatic breast cancer, with ER and PR positive but HER-2 negative. She was managed with a selective estrogen receptor degrader and $C D K 4 / 6$ inhibitor and has been in remission since. Metastasis to the small bowel from the breast is a very rare occurrence. Clinicians should thus maintain a modest amount of suspicion when encountering an uncommon GI presentation of primary breast malignancy. We describe the case of metastatic breast cancer with an atypical GI presentation.
\end{abstract}

Categories: Radiology, Gastroenterology, Oncology

Keywords: breast cancer, metastasis, small bowel obstruction, iron deficiency anemia, gastrointestinal tract

\section{Introduction}

Breast cancer accounts for $30 \%$ of all new cancer cases in women each year in the US, and it has a yearly mortality rate of $14 \%$ [1]. Among invasive breast carcinomas, invasive lobular cancer forms the second most common type with an incidence rate of 5-15\% [2]. Invasive lobular carcinomas are characteristically multifocal when present in the unilateral breast, but more often they are present bilaterally [3].

Out of the diagnosed breast cancers, about 5-10\% leads to metastasis, and at least $20-50 \%$ of patients develop metastasis at least once in their lifetime [4]. There has been a drastic increase in the survival rate of patients with breast cancer owing to timely recognition and prompt management. However, metastasis still occurs in $30 \%$ of the patients even after treatment with hormonal therapy, chemotherapy, radiotherapy, and surgical intervention [5]. Prognostic factors include the size and grade of the primary diagnosis, regional lymph node involvement, presence of hormonal receptors, and metastatic site involvement [6]. Breast cancer frequently metastasizes to the liver, lungs, brain, adrenals, and, very rarely, the bones. Metastasis to the gastrointestinal (GI) tract is atypical, although involvement can occur anywhere from the oropharynx to the anus [7]. We describe the case of an elderly female with GI metastasis, masked as small bowel obstruction, from primary breast cancer.

\section{Case Presentation}

A 72-year-old Caucasian female with a past medical history of bilateral lobular breast carcinoma, paroxysmal atrial fibrillation, and hyperthyroidism presented with an intermittent history of nausea and vomiting leading to decreased oral intake, diarrhea, intermittent abdominal pain, and unintentional weight loss of approximately 30-40 lb. The patient complained of an average of three bowel movements per day, more diarrhea than constipation with no change in consistency and absence of blood and mucus along with generalized abdominal pain, which was cramping in nature and got aggravated after meals. She denied heartburn or consuming any particular food that caused nausea.

The patient had been diagnosed with lobular carcinoma of the right breast 24 years ago. She had been managed with partial mastectomy, radiation, and adjuvant chemotherapy including fluorouracil, methotrexate, and cyclophosphamide followed by nine years of hormonal therapy with tamoxifen. The patient had been further diagnosed with lobular carcinoma of the left breast approximately 10 years after the initial diagnosis. She had undergone lumpectomy and had completed a five-year course of hormonebased chemotherapy with anastrozole. The patient had since been in remission. 


\section{Cureus}

that time had revealed a hemoglobin of $6.5 \mathrm{gm} / \mathrm{dl}$ (normal range: 11.7-15.1 gm/dl), hematocrit of $21 \%$ (normal range: 29.4-47.0\%), mean corpuscular volume (MCV) of $88 \mathrm{fl}$ (normal range: 78.9-98.6 fl), and positive fecal occult blood. Iron studies indicated iron of $10 \mathrm{mcg} / \mathrm{dl}$ (normal range: $50-212 \mathrm{mcg} / \mathrm{dl}$ ), a total iron-binding capacity of $381 \mathrm{mcg} / \mathrm{dl}$ (normal range: $250-450 \mathrm{mcg} / \mathrm{dl}$ ), transferrin saturation of $3 \%$ (normal range: $15-50 \%$ ) and ferritin of $6.8 \mathrm{ng} / \mathrm{ml}$ (normal range: $11-307 \mathrm{ng} / \mathrm{ml}$ ). Imaging studies including CT of the abdomen had been unremarkable for any acute abnormality. Esophagogastroduodenoscopy (EGD) and colonoscopy showed mild gastritis and ulcer in the sigmoid colon along with internal and external hemorrhoids. The biopsy of the gastric ulcer had revealed no malignant changes. She had been managed with proton-pump inhibitor and blood transfusion. The patient had received parenteral iron transfusions as an outpatient.

On arrival to the emergency department, she had a pulse of 58 beats/minute, blood pressure of $98 / 46 \mathrm{~mm}$

$\mathrm{Hg}$, oxygen saturation of $99 \%$ on room air, and body mass index of $19.5 \mathrm{~kg} / \mathrm{m}^{2}$. A physical examination showed pallor. Abdominal examination was significant for distension along with tenderness in the left lower quadrant. Laboratory investigations were noteworthy for hemoglobin of $8.2 \mathrm{gm} / \mathrm{dl}$, hematocrit of $24 \%$, MCV of $89 \mathrm{fl}$, red cell distribution width of $16.8 \%$ (normal range: $11.5-15.5 \%$ ). The hepatic panel revealed aspartate aminotransferase of $190 \mathrm{u} / 1$ (normal range: 13-39 u/l), alanine transaminase of $157 \mathrm{u} / \mathrm{l}$ (normal range: 7-52 u/l), and alkaline phosphatase of $27 \mathrm{u} / \mathrm{l}$ (normal range: 34-104 $\mathrm{u} / \mathrm{l}$ ). Further workup revealed Ca125 of $93.6 \mathrm{u} / \mathrm{ml}$ (normal range: $0-35 \mathrm{u} / \mathrm{ml}$ ) and carcinoembryonic antigen (CEA) of $12.6 \mathrm{u} / \mathrm{ml}$ (normal range: 0.0-3.0 u/ml). Imaging studies of the abdomen including radiograph, MRI with contrast (Figure 1), and CT scan (Figure 2) confirmed the presence of distal small bowel obstruction. The patient underwent small-bowel resection along with primary anastomosis after the detection of a small bowel tumor.

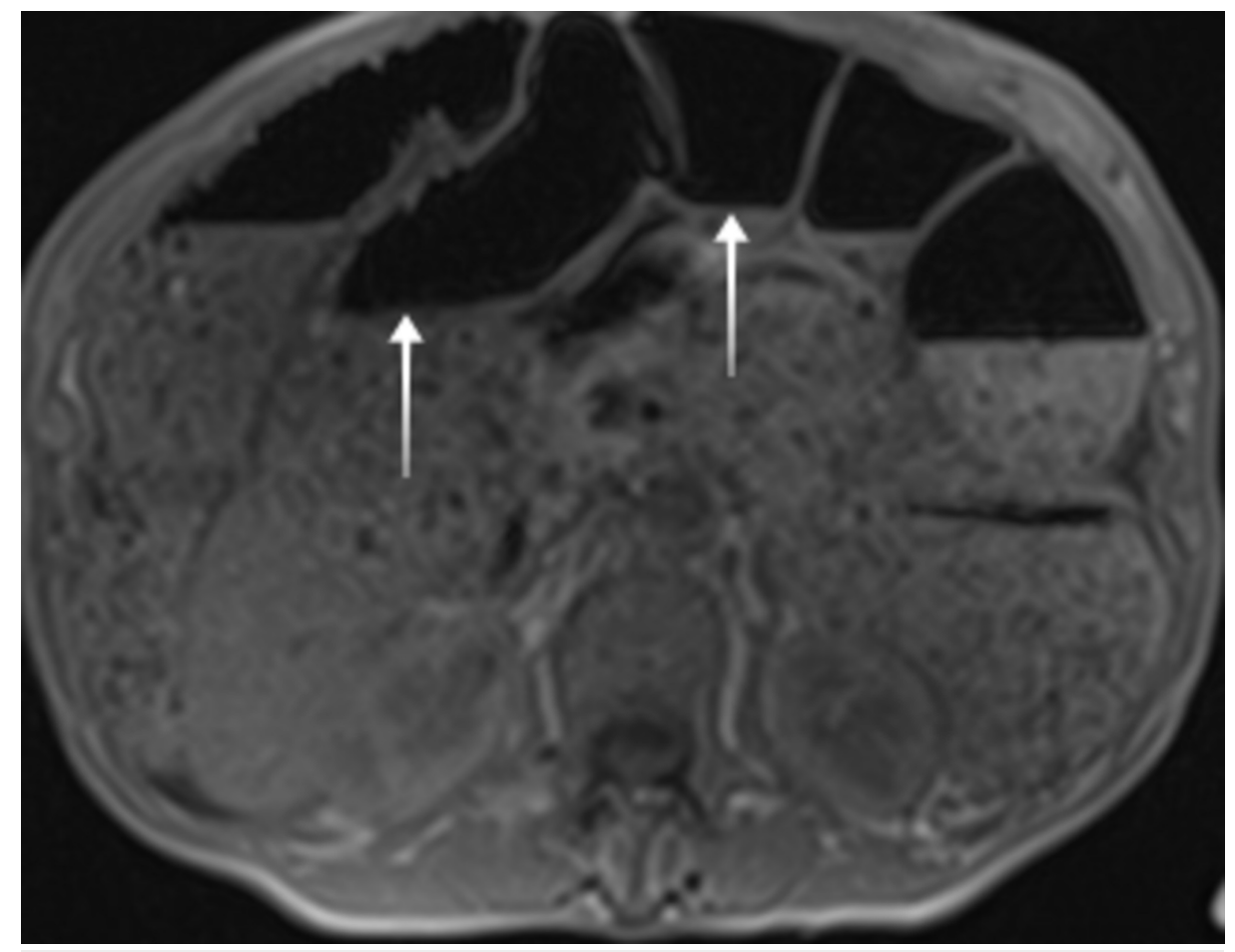

FIGURE 1: MRI of the abdomen showing distention of large intestine and small bowel with air-fluid levels concerning for distal obstruction.

MRI: magnetic resonance imaging 


\section{Cureus}

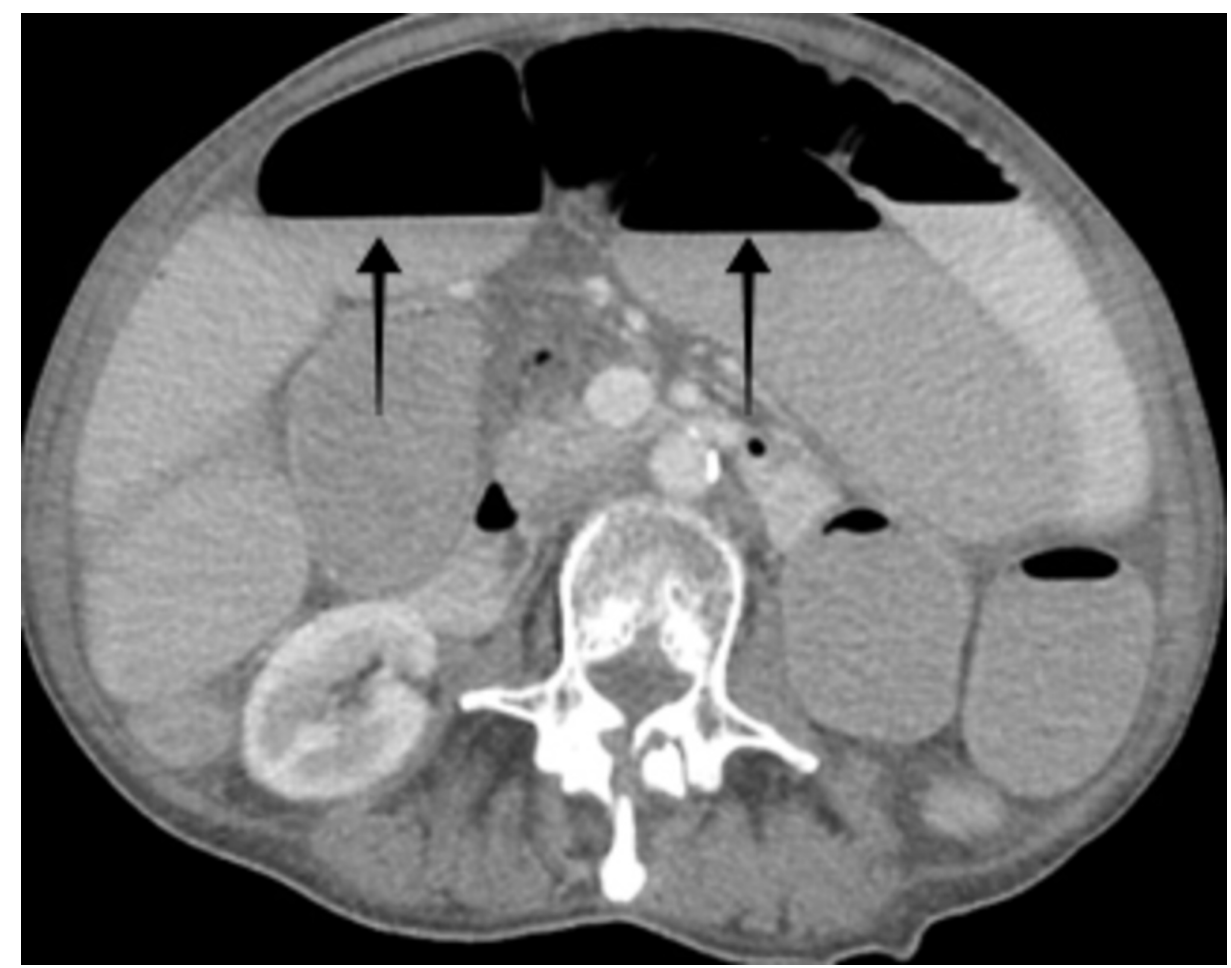

FIGURE 2: CT scan of the abdomen revealing high-grade small bowel obstruction with transition point noted centrally in the pelvis in the region of thickened small bowel loops.

CT: computed tomography

Histopathology showed the presence of metastatic pleomorphic lobular carcinoma consistent with breast primary (Figure 3).

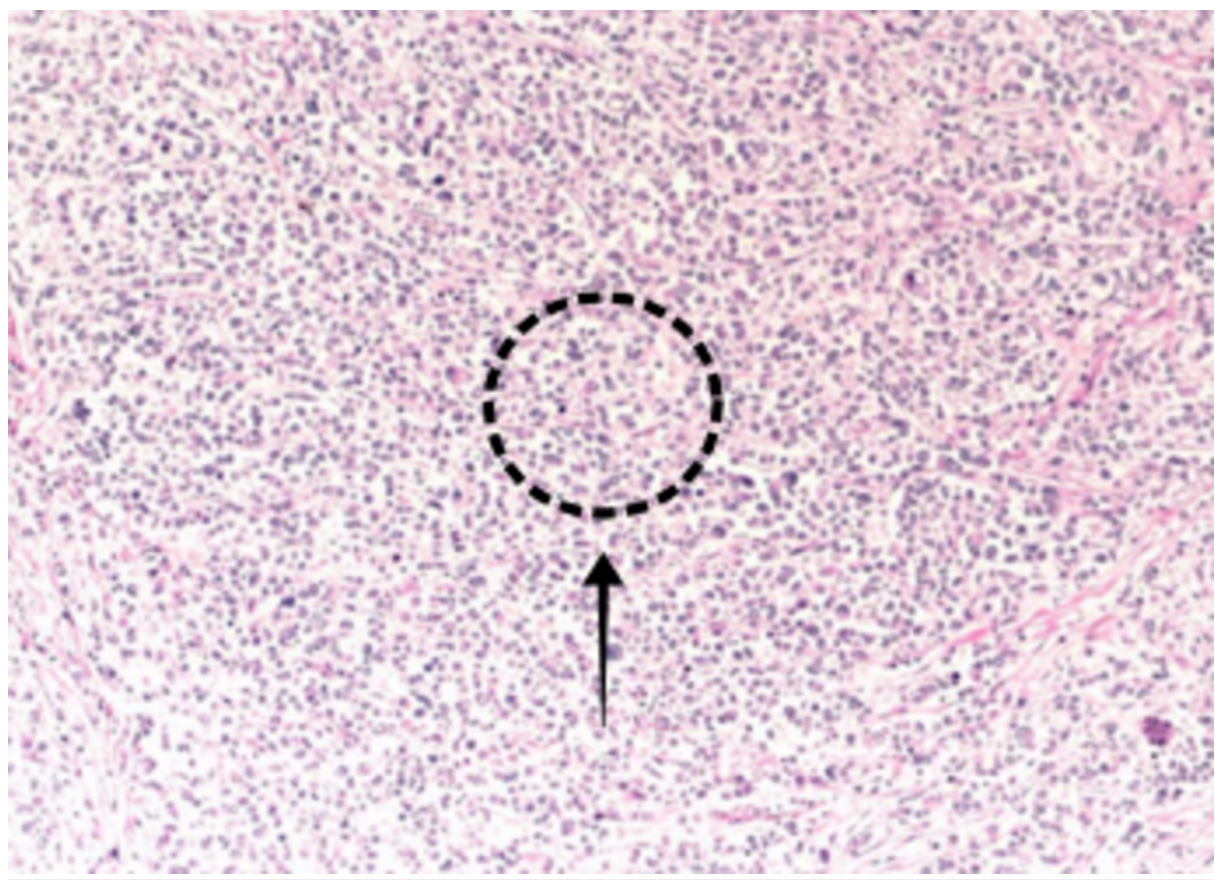

FIGURE 3: Biopsy specimen of resected small bowel showing multifocal metastatic pleomorphic lobular carcinoma present, consistent with breast primary. It shows positive mesenteric margin and negative 


\section{Cureus}

proximal and distal surgical resection margins.

Further workup showed cytoplasmic staining with p120 catenin and positive for GATA-binding protein 3 (GATA-3) (Figure 4), focally positive for both estrogen receptor (ER) and progesterone receptor (PR), gross cystic disease fluid protein 15 (GCDFP-15) and mammaglobin, but negative for human epidermal growth factor receptor 2 (HER-2).

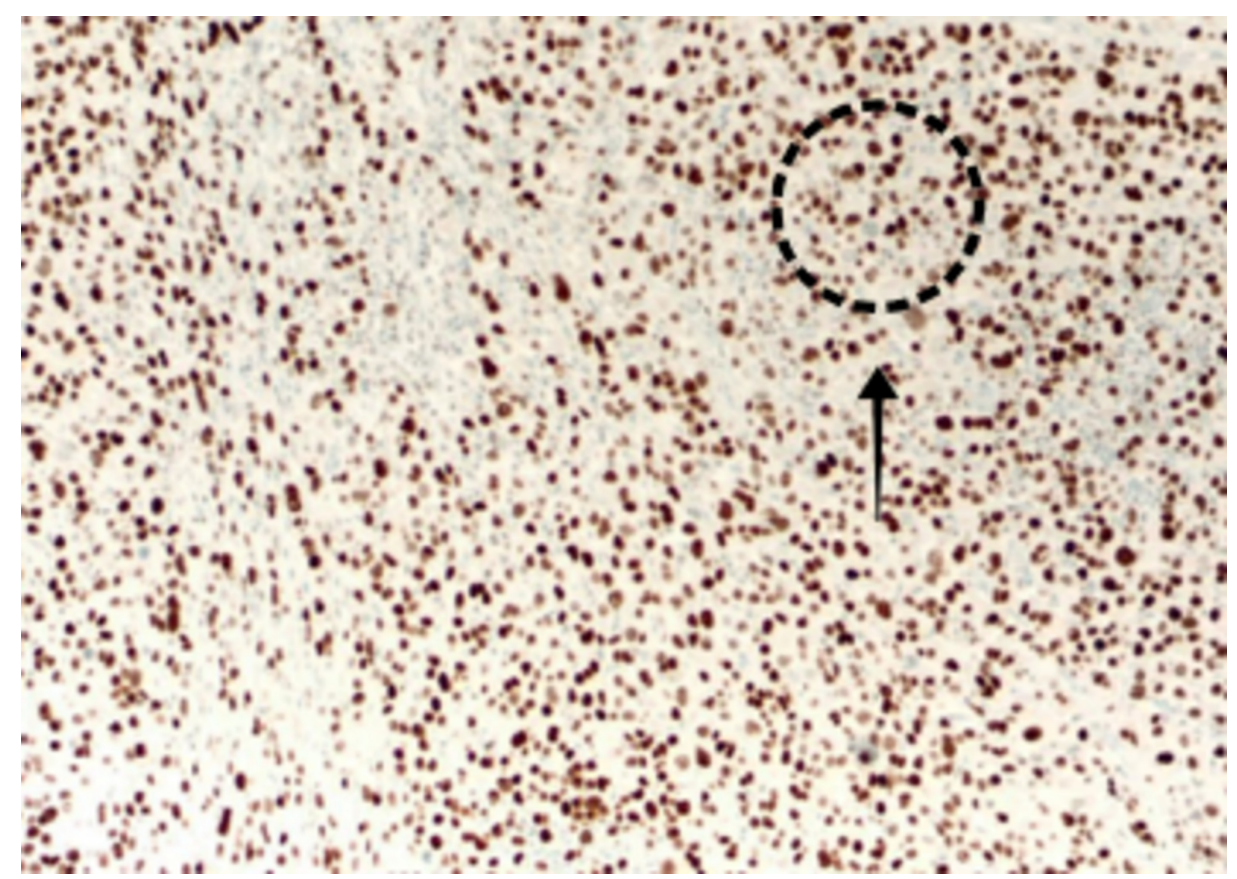

FIGURE 4: GATA 3+ stain.

GATA3: GATA-binding protein 3

Two lymph nodes near the tumor site were identified, out of which one demonstrated the presence of metastatic carcinoma (Figure 5).

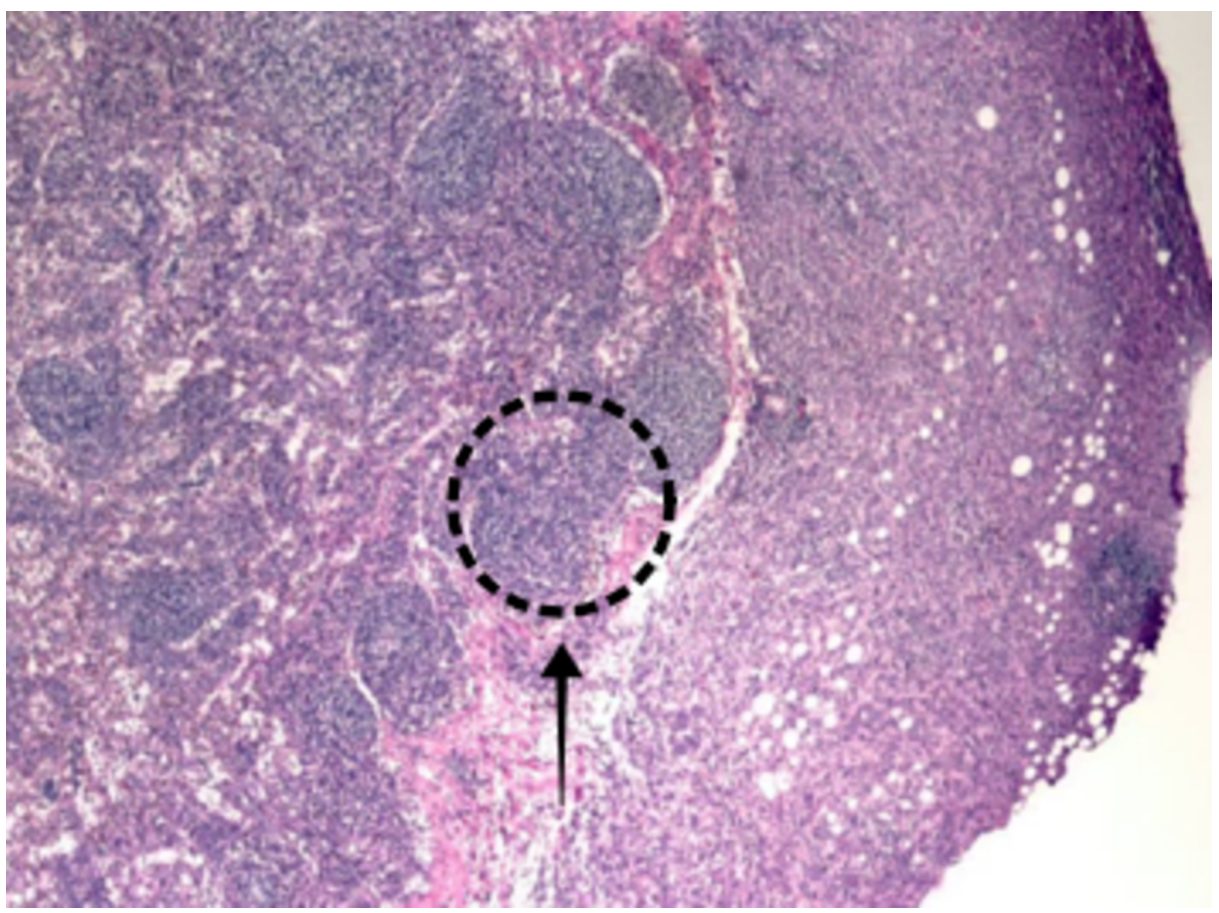


The patient was subsequently started on fulvestrant and palbociclib. She has shown significant improvement in her symptoms.

\section{Discussion}

Small bowel malignancies comprise about 1-3\% of primary GI cancers [8]. Small bowel metastasis is commonly seen in primary malignancies like colon cancer, malignant melanoma, and cervical cancer [9]. The involvement of the GI tract in primary breast cancer is a rare occurrence. Borst et al. reported that out of 2,500 cases of metastatic breast cancer they studied, only 17 cases (less than $1 \%$ ) were found to have GI metastasis [10]. Stomach and small bowel metastasis are seen more often when compared to metastasis to the colon and rectum.

Lobular breast cancer metastasizes in a particular pattern and more commonly involves the GI tract and the retroperitoneum, even though infiltrative ductal breast carcinoma is more prevalent [11]. Invasive lobular cancer occurs more commonly in postmenopausal females, postulated to be due to hormone replacement therapy [12]. Our patient presented with bilateral lobular breast carcinoma that had metastasized to the small bowel and manifested with symptoms of obstruction.

Primary GI tumors are commonly positive for cytokeratin (CK) 20 and CEA, which is not seen in breast cancers. On the other hand, the metastatic lobular carcinoma is routinely positive for ER, PR, CK 7, and GCDFP-15 whereas it is negative for vimentin $[13,14]$. PR-positive breast cancers have a greater predisposition of GI metastasis when compared to ER-positive tumors [15]. Our patient was positive for CEA, ER and PR, and GCDFP-15, but negative for HER-2 tumor markers.

Previous studies have shown varying time durations between the diagnosis of primary breast cancer and the detection of GI metastasis. McLemore et al. revealed that the mean time interval between primary breast cancer and manifestations of metastatic cancer was seven years, with the median age of primary diagnosis being 55 years [16]. However, a literature search yielded an incidence of gastric metastasis 30 years after the primary diagnosis of breast cancer [17]. The present case shows GI metastasis after 24 years of the diagnosis of primary cancer.

The presentation of GI metastases ranges from no symptoms to manifestations of serious obstruction. As reported by McLemore et al., the frequent manifestations were abdominal pain, melena, nausea and vomiting, difficulty in swallowing, fatigue, weight loss, and a palpable mass [16]. Our patient presented with intermittent abdominal pain, unintentional weight loss, and severe iron-deficiency anemia.

There is no consensus in the medical fraternity about the management of breast cancer metastasizing to the GI tract. Several treatment modalities including systemic chemotherapy, hormonal therapy, and surgical procedure are available. Symptoms like intestinal obstruction, hemorrhage, or perforation necessitate surgical involvement. McLemore et al. reported in their study that GI metastatic patients who underwent palliative surgery had a longer median survival (44 months vs. nine months); however, it was statistically insignificant [16]. The study revealed that the average survival of metastasis to the GI tract from primary breast cancer was 28 months, with chemotherapy and tamoxifen treatment having a significant influence [16]. Tang et al. studied about two patients with metastatic lobular cancer presenting as intestinal obstruction who were treated with fulvestrant, a parenteral estrogen receptor antagonist [18]. Both cases showed the resolution of intestinal manifestations after fulvestrant administration. Our patient underwent resection of the small bowel tumor and was treated with selective estrogen receptor degrader, fulvestrant, and the CDK4/6 inhibitor, palbociclib.

\section{Conclusions}

Instances of patients with primary breast cancer presenting with GI metastasis are uncommon and peculiar. These circumstances illustrate the importance of keeping GI metastasis as a likely differential, and physicians should be vigilant about patients with breast cancer, especially of the lobular type, who present with unusual GI manifestations.

\section{Additional Information}

\section{Disclosures}

Human subjects: Consent was obtained by all participants in this study. UPMC Pinnacle Institutional Review Board issued approval NA. As long as no personal identifiable information is used and all information presented for publication is de-identified per HIPAA regulations, this research is exempt under 45 CFR 46.101 (b) (4) and does not need to be submitted to the IRB for review and approval. Conflicts of interest: In compliance with the ICMJE uniform disclosure form, all authors declare the following: 
Payment/services info: All authors have declared that no financial support was received from any organization for the submitted work. Financial relationships: All authors have declared that they have no financial relationships at present or within the previous three years with any organizations that might have an interest in the submitted work. Other relationships: All authors have declared that there are no other relationships or activities that could appear to have influenced the submitted work.

\section{References}

1. Siegel RL, Miller KD, Jemal A: Cancer statistics, 2018. CA Cancer J Clin. 2018, 68:7-30. 10.3322/caac.21442

2. Rakha EA, El-Sayed ME, Menon S, Green AR, Lee AH, Ellis IO: Histologic grading is an independent prognostic factor in invasive lobular carcinoma of the breast. Breast Cancer Res Treat. 2008, 111:121-127. 10.1007/s10549-007-9768-4

3. Arrangoiz R, Papavasiliou P, Dushkin H, Farma JM: Case report and literature review: metastatic lobular carcinoma of the breast an unusual presentation. Int J Surg Case Rep. 2011, 2:301-305. Accessed: November 19, 2019: 10.1016/i.ijscr.2011.06.010

4. Cardoso F, Harbeck N, Fallowfield L, Kyriakides S, Senkus E; ESMO Guidelines Working Group: Locally recurrent or metastatic breast cancer: ESMO clinical practice guidelines for diagnosis, treatment and followup. Ann Oncol. 2012, 23:11-19. 10.1093/annonc/mds232

5. Eljabu W, Finch G, Nottingham J, Vaingankar N: Metastatic deposits of breast lobular carcinoma to small bowel and rectum. Int I Breast Cancer. 2011, 2011: 10.4061/2011/413949

6. Mouawad NJ, Cleary RK: Small bowel obstruction as the primary presentation of undiagnosed metastatic lobular breast carcinoma. Breast Dis. 2011, 33:35-40. 10.3233/bd-2010-0317

7. Ambroggi M, Stroppa EM, Mordenti P, et al.: Metastatic breast cancer to the gastrointestinal tract: report of five cases and review of the literature. Int J Breast Cancer. 2012, 2012:439023. 10.1155/2012/439023

8. Johnston CA, Yung DE, Joshi A, Plevris JN, Koulaouzidis A: Small bowel malignancy in patients undergoing capsule endoscopy at a tertiary care academic center: case series and review of the literature. Endosc Int Open. 2017, 5:E463-470. 10.1055/s-0043-106186

9. Choi JE, Park SY, Jeon MH, Kang SH, Lee SJ, Bae YK, Kim MK: Solitary small bowel metastasis from breast cancer. J Breast Cancer. 2011, 14:69-71. 10.4048/jbc.2011.14.1.69

10. Borst MJ, Ingold JA: Metastatic patterns of invasive lobular versus invasive ductal carcinoma of the breast . Surgery. 1993, 114:637-641.

11. Théraux J, Bretagnol F, Guedj N, Cazals-Hatem D, Panis Y: Colorectal breast carcinoma metastasis diagnosed as an obstructive colonic primary tumor. A case report and review of the literature. Gastroenterol Clin Biol. 2009, 33:1114-1117. 10.1016/j.gcb.2009.05.015

12. Douvetzemis SE, Kokkinos C: Small bowel obstruction due to metastatic breast cancer . Hellenic Journal of Surgery. 2015, 87:419-422. 10.1007//13126-015-0249-7

13. Hsieh PS, Yeh CY, Chen JR, Changchien CR: Ileocecal breast carcinoma metastasis. Int J Colorectal Dis. 2004, 19:607-608. 10.1007/s00384-004-0612-6

14. Tot T: The role of cytokeratins 20 and 7 and estrogen receptor analysis in separation of metastatic lobular carcinoma of the breast and metastatic signet ring cell carcinoma of the gastrointestinal tract. APMIS. 2000, 108:467-472. 10.1034/i.1600-0463.2000.d01-84.x

15. Schwarz RE, Klimstra DS, Turnbull AD: Metastatic breast cancer masquerading as gastrointestinal primary . Am J Gastroenterol. 1998, 93:111-114. 10.1111/j.1572-0241.1998.111 c.x

16. McLemore EC, Pockaj BA, Reynolds C, Gray RJ, Hernandez JL, Grant CS, Donohue JH: Breast cancer: presentation and intervention in women with gastrointestinal metastasis and carcinomatosis. Ann Surg Oncol. 2005, 12:886-894. 10.1245/aso.2005.03.030

17. Benfiguig A, Anciaux ML, Eugène CI, Benkémoun G, Etienne JC: Gastric metastasis of breast cancer occurring after a cancer-free interval of 30 years (Article in French). Ann Gastroenterol Hepatol (Paris). 1992, 28:175-177.

18. Tang JY, Rampaul RS, Cheung KL: The use of fulvestrant, a parenteral endocrine agent, in intestinal obstruction due to metastatic lobular breast carcinoma. World J Surg Oncol. 2008, 6:128. Accessed: November 19, 2019: 10.1186/1477-7819-6-128 